\title{
Protée
}

\section{Peirce et la limite}

\section{l'adresse nécessaire du signe}

\section{Nelson Charest}

Volume 33, numéro 1, printemps 2005

L’allégorie visuelle

URI : https://id.erudit.org/iderudit/012271ar

DOI : https://doi.org/10.7202/012271ar

Aller au sommaire du numéro

\section{Éditeur(s)}

Département des arts et lettres - Université du Québec à Chicoutimi

ISSN

0300-3523 (imprimé)

1708-2307 (numérique)

Découvrir la revue

Citer cet article

Charest, N. (2005). Peirce et la limite : l'adresse nécessaire du signe. Protée, 33(1), 103-111. https://doi.org/10.7202/012271ar

\section{Résumé de l'article}

Cet article veut montrer que le signe peircien, par son adresse nécessaire, rejoint les pratiques de l'herméneutique. Nous procédons, dans un premier temps, à une révision des Catégories peirciennes, en notant comment cette pensée prépare les définitions du signe. Puis nous évaluons quelques-unes de ces définitions, et nous les complétons par des remarques sur la semiosis, l'habitude et la croyance, pour enfin établir un lien entre le signe peircien et l'herméneutique, selon trois aspects : la polysémie, l'autorité et l'adresse.
Ce document est protégé par la loi sur le droit d'auteur. L'utilisation des services d'Érudit (y compris la reproduction) est assujettie à sa politique d'utilisation que vous pouvez consulter en ligne.

https://apropos.erudit.org/fr/usagers/politique-dutilisation/ 


\title{
PEIRCE ET LA LIMITE: L'ADRESSE NÉCESSAIRE DU SIGNE
}

\author{
NELSON ChAREST
}

La sémiotique peircienne semble devoir s'opposer à l'herméneutique pour en montrer l'insuffisance. Dans son ouvrage sur la Pragmatique de la signification, Jean Fisette prend I'herméneutique à contrepartie, sur deux points majeurs, pour mieux situer la pensée de Peirce: 1. la cohérence interne de l'œuvre, selon lui illusoire, et relevant plutôt du travail d'interprétation (qu'il oppose, en ce sens, à l'interprétance); 2. le sens originel de l'œuvre, tout aussi utopique selon Fisette, puisque le signe est immédiatement transporté par la semiosis et qu'un « texte est un processus de sortie de lui-même» (Fisette, 1996: 32)1 ${ }^{1}$, pour reprendre sa belle expression. Or, ces deux aspects sont soutenus par l'herméneutique elle-même, il est vrai, dans ses développements encore récents. Ainsi, Michel Charles pense que la cohérence n'est pas donnée par le texte, mais qu'elle est construite par l'analyse ${ }^{2}$. Quant au sens originel, Gadamer lui-même le pense utopique, et pousse d'ailleurs plus loin: le sens de I'œuvre n'est pas à chercher dans son origine, mais plutôt dans ses développements, car le propre de l'œuvre esthétique (son «être») est précisément cette «sortie hors de soi» dont parle Fisette $^{3}$.

$C^{\prime}$ est ce dernier aspect qui nous intéresse ici, ce qu'on appellera la «désadhérence» du signe. Pour reprendre un titre d'Eco, nous ne cherchons pas une "limite de l'interprétation», mais, en deçà, une limite du signe - cette «porte de sortie» que le signe emprunte lorsqu'il entre dans la re-présentation. À ce point, nous tenterons de le démontrer, la sémiotique peircienne et l'herméneutique se rejoignent et peuvent s'informer l'une I'autre. Cette limite, nous pensons pouvoir la nommer l'adresse du signe, en prenant acte de la polysémie du terme: 1 . indication du nom et du domicile d'une personne; 2. signe sous lequel est classée une information; mais aussi: 1. qualité physique d'une personne qui fait les mouvements les mieux adaptés à la réussite de l'opération; et 2. qualité d'une personne qui sait s'y prendre, manœuvrer comme il faut pour obtenir un résultat. On aura encore en tête ces trois autres aspects: 1 . que le verbe «adresser», dans toutes ses acceptions, suppose «quelqu'un», qui est le récepteur de l'action du verbe; 2 . que le mot a donné lieu à I'expression «double adresse», qui suppose une médiation, comprise aussi dans le signe peircien; et 3. que l'étymon du terme est la « direction », idée incluse dans toutes les définitions précitées, et qui nous semble réactiver avec bonheur la polysémie d'un autre terme fortement apparenté, celle du «sens». Pour arriver à mieux cerner cette limite du signe, nous effectuons d'abord une lecture «herméneutique» du texte peircien, lecture naïve si l'on veut, puisqu'elle ne s'attarde qu'à quelques notions élémentaires de cette pensée riche (les Catégories, le Signe). Ensuite, nous situons la limite, «l'adresse» du signe dans l'interprétant, que nous tentons de définir comme le lieu où le signe se re-présente ${ }^{4}$. Enfin, nous reprenons l'étude du signe d'un point de vue herméneutique, en tentant de montrer comment la «lecture » (l'interprétance) du signe est essentielle à sa survie et, inversement, comment la juste compréhension du signe peircien passe par une prise en compte de sa lecture; nécessité, donc, du croisement entre sémiotique et herméneutique.

\section{Retour sur Peirce}

La pensée de Peirce est, dès le départ, celle d'un croisement; elle est tantôt une logique, tantôt une pragmatique ou plutôt un "pragmaticisme», tantôt une sémiotique. Lui-même préfère parler de "phanéroscopie» (du grec phaneron, «ce qui se 
montre», phénomène), mais il endosse aussi les autres termes. Ce jeu viserait peut-être, au plus simple, à rendre ceci: la pensée se construit de l'extérieur. Peirce ne croit pas à une pensée autonome, intérieure, indépendante des perceptions, et ne croit d'ailleurs pas plus à la possibilité de toute introspection, ni d'une conscience interne ${ }^{5}$. La pensée s'élabore à partir de l'extérieur, et y retourne. Le "phanéron", en tant qu'il se montre, frappe la perception. Il le fait par sa qualité d'être un signe, qui seule permet le développement d'une pensée (passage de la perception au jugement sur cette perception $)^{6}$. Et le signe, après avoir traversé la pensée, se forme en un interprétant final, qui est une habitude développée dans la réalité. Nous aurions donc: phanéroscopie $\rightarrow$ sémiotique $->$ pragmatique. En devançant un peu notre développement, nous considérerons que le moyen terme, parce qu'il est relationnel, subsume les deux autres et peut donc, dans sa pleine extension, en rendre compte. Optons donc pour la sémiotique.

\subsection{CATÉGORIES}

Nous commencerons cette enquête par une remarque qui paraîtra incidente, mais qui au contraire nous semble primordiale:

Quant à moi, je ne suis pas l'ennemi déclaré d'un nombre innocent; je respecte et estime tous les nombres pour ce qu'ils sont; mais je suis forcé d'avouer qu'en philosophie, j'ai un penchant marqué pour le nombre Trois. En fait, j'utilise si couramment la division trichotomique dans mes spéculations qu'il me semble préférable de commencer par une brève étude préliminaire des conceptions sur lesquelles ces divisions doivent reposer. Je n'entends rien de plus que les idées de premier, second, troisième - idées si vastes qu'on peut les regarder plutôt comme des dispositions ou des tons de la pensée que comme des notions définies, mais qui, de ce fait, ont une grande portée. (Peirce, 1978: 71-72)

Avant d'aller plus avant dans la compréhension de cette remarque, considérons encore cet autre extrait, qui la recoupe:

Les catégories cénopythagoriciennes constituent évidemment un nouvel essai de caractérisation de ce que Hegel a essayé de caractériser par ses trois moments de la pensée. Elles correspondent aussi aux trois catégories de chacune des quatre triades de la table de Kant. Mais le fait que ces différentes tentatives furent faites indépendamment les unes des autres [...] n'aboutit qu'à montrer qu'il n'y a vraiment que ces trois éléments. (Ibid.: 23)

Dans ces deux extraits, Peirce défend un nombre, le trois, ce qui produira dans son système la tiercéité et les trichotomies. Or, ce sur quoi nous voulons insister est qu'à deux reprises, il défend le trois contre ce qui pourrait être davantage: «Je n'entends rien de plus [...]», «idées si vastes [...]», «il n'y a vraiment que ces trois éléments ». Ce que Peirce cherche à montrer est qu'aussi vaste que soit le monde à étudier, il se réduit toujours à une trichotomie, et rien de plus. Or, de notre point de vue à nous modernes, Peirce est bien plutôt celui qui a augmenté le signe saussurien 7 .

La base de la pensée peircienne se compose donc des trois Catégories suivantes: Priméité, Secondéité, Tiercéité. Avant de les décrire, remarquons tout de suite ce que ces dénominations révèlent concernant leur nombre: Priméité, en tant que premier; Secondéité, en tant que second (et non «deuxième», et donc en réaction au premier); Tiercéité, en tant que tiers (et non «troisième», et donc mitoyen dans une relation du premier au second $^{8}$ ). On notera également, avant $d^{\prime}$ en expliquer les raisons, que Peirce commence habituellement ses descriptions par le second. De là, il remonte au premier, par le moyen du tiers.

Que sont donc ces catégories?

Mon opinion est qu'il y a trois modes d'être. Je soutiens que nous pouvons les observer directement dans les éléments de tout ce qui est à n'importe quel moment présent à l'esprit d'une façon ou d'une autre. Ce sont l'être de la possibilité qualitative positive, l'être du fait actuel, et l'être de la loi qui gouverne les faits dans le futur.

(Peirce, 1978: 69)

Le premier est ce dont l'être est simplement en soi; il ne renvoie à rien et n'est impliqué par rien. Le second est ce qui est ce qu'il est en vertu de quelque chose, par rapport à quoi il est second. Le troisième est ce qui est ce qu'il est par les choses entre lesquelles il établit un lien et qu'il met en relation. (Ibid.: 72)

La Catégorie Première est l'Idée de ce qui est tel qu'il est indépendamment de quoi que ce soit d'autre. En d'autres termes, c'est une Qualité de Sentiment. [...] La Catégorie Deuxième est I'ldée de ce qui est tel qu'il est en étant deuxième relativement à quelque Premier, indépendamment de quoi que ce soit d'autre, et en particulier indépendamment de n'importe quelle loi, bien qu'il puisse se conformer à une loi. En d'autres termes, c'est la Réaction, en tant qu'élément du phénomène. [...] Une simple complication de la Catégorie Troisième, n'impliquant aucune idée essentiellement différente, donnera l'idée de quelque chose qui est tel qu'il est en vertu de ses relations à n'importe quelle multitude finie, dénombrable, ou non dénombrable ou même à n'importe quelle supermultitude de corrélats; en sorte que cette catégorie suffit à elle seule à donner la conception de la Vraie Continuité, à laquelle aucune conception jusqu'ici découverte n'est supérieure. (Peirce, 2002 : 305-306) 
Le premier est une Qualité sans adhérence qui précède toute relation, une "possibilité qualitative positive». Peirce emprunte, pour l'expliquer, l'exemple d'une couleur, disons le rouge. II existe du rouge en soi, que l'on doit penser avant de l'appliquer à un quelconque objet; dire «cette robe rouge» n'est donc plus une Priméité, mais un « fait», donc un second. Il existe une qualité commune entre «cette robe rouge» et «ce vin rouge», ou entre "cette robe rouge » et "cette autre robe rouge», qualité commune qui nous oblige à poser l'existence de la Priméité. Elle est donc inexprimable en soi, puisqu'en l'évoquant nous établissons d'emblée avec elle une relation (réaction) qui est de l'ordre de la secondéité. Il faudrait quand même noter que, probablement à cause justement de cette impossibilité, la poésie, particulièrement, semble par moments attachée à saisir cette Priméité, en tant que premier. Il me semble que c'est là une partie de la quête de Ponge, et par le fait même de son «échec», de sa « rage de l'expression ». En ce sens, Michel Collot a raison de proposer le syntagme de «matière-émotion » : la matière ne se donne jamais en elle-même, comme pure qualité (le froid, le rugueux, le doux, etc.); mais la matière-émotion représente peut-être le dernier stade (le stade le plus «reculé») d'une quête de la Priméité 9 .

Le second se définit en termes de Réaction - ailleurs Peirce dira même Lutte, et s'applique au «fait actuel ». Il est ce qu'il est en tant qu'il entre en relation avec un premier. Si Peirce associe le fait à la Réaction, comme second, c'est que, dans le fait, la Qualité est adhérente et qu'elle est donc, à la base, en relation avec un objet; il y a interaction, «lutte» entre les deux, et c'est ce qui produit le «fait». Peirce exclut la «loi » du second car, en tant qu'il se définit par son rapport à un premier, le second est toujours «relatif». Sa seule régularité provient de cette Réaction elle-même, qui le définit dans son actualité.

Le troisième est la pensée qui gouverne le signe du second, comme réaction à un premier. Il est une loi en ce qu'il détermine la continuité qui règle la réaction du second au premier. En établissant une loi, le troisième ramène la multitude à une unité et rend compte de relations qui sont vérifiables tant pour le passé que pour le futur; la loi possède cette ubiquité d'être transposable ici et là simultanément. Il est donc toujours un tiers qui subsume les deux premières Catégories et qui règle leurs rapports ${ }^{10}$. Et $c^{\prime}$ est parce qu'il est tiers qu'il est la dernière catégorie: la multitude des faits, aussi nombreux soient-ils, peut toujours être ramenée à une loi qui en montre la continuité. Si un fait rebelle vient à s'écarter de cette loi, c'est qu'il est soumis à une autre loi ; et cette loi pourra encore entrer en relation avec la première, jusqu'à ce qu'on trouve la tierce loi qui les gouverne toutes deux.
C'est là, si l'on veut, l'élégance et l'économie de la pensée de Peirce.

\subsection{SIGNE ET INTERPRÉTANT}

Le signe peircien découle directement de ces trois Catégories, ce qui lui donne son énorme extension. II n'y a pas ici distinction entre fiction et vérité, entre discours et expérience, entre figure et référence. Dans la mesure où la pensée s'élabore toujours par signes, Peirce leur donne une extension maximale. Est signe ce qui répond à ses définitions du signe. Il en a fourni plusieurs; nous en donnons un échantillon, que nous discutons au fur et à mesure:

1. [Un] signe a comme tel trois références : en premier lieu, c'est un signe pour quelque pensée qui l'interprète; en deuxième lieu, c'est un signe mis pour un objet auquel pour cette pensée il est équivalent; en troisième lieu, c'est un signe, sous quelque aspect ou qualité, qui le met en liaison avec son objet. (Peirce, 2002: 51)

Cette première définition montre le lien entre le signe et les Catégories. En tant que définition, elle est une loi qui ressortit à la Tiercéité; mais elle intègre elle aussi une trichotomie qui imite celle des Catégories ${ }^{11}$. On notera que Peirce inverse l'ordre, de la «pensée» (Tiercéité) à l'objet (Secondéité), puis à la qualité (Priméité). On remarquera aussi que la qualité a ici valeur de «liaison», ce qui résulte justement de l'inversion de l'ordre: en accédant à la qualité par l'objet, on est à même de percevoir sur elle la réaction d'un fait, et donc une liaison qu'elle n'a pas en elle-même.

2. Un signe, ou representamen, est quelque chose qui tient lieu pour quelqu'un de quelque chose sous quelque rapport ou à quelque titre. Il s'adresse à quelqu'un, c'est-à-dire crée dans l'esprit de cette personne un signe équivalent ou peut-être un signe plus développé. Ce signe qu'il crée, je l'appelle l'interprétant du premier signe. Ce signe tient lieu de quelque chose: de son objet. II tient lieu de cet objet, non sous tous rapports, mais par référence à une sorte d'idée que j'ai appelée quelquefois le fondement du representamen.

(Peirce, 1978: 121)

Cette définition du signe est la plus connue et la plus complète. Elle donne lieu à plusieurs remarques, et dès son amorce, avec cette ambivalence entre «signe» et «representamen». II semblerait, au premier abord, y avoir confusion entre le tout et la partie; le signe semble pouvoir devenir une partie de lui-même (signe = signe - interprétant - objet), d'où justement la nécessité de proposer le terme de «representamen». Or, il y a au moins 
une raison de conserver cette ambiguïté ou de la noter: le representamen, en tant qu'il frappe la perception, est le véritable « signe » qui accède à la pensée. L'interprétant, pour sa part, constitue le mouvement, le tiers qui permet la liaison; et l'objet est la chose indiquée par le signe, qui en tient lieu et en quelque sorte le remplace. Or il existe des liaisons erronées, et aussi des objets qui ne peuvent «signifier » (ce qui revient à un échec de liaison), car, comme Peirce le note, l'objet doit lui-même être un signe:

Tout signe est mis pour un objet indépendant de lui-même; mais il ne peut être un signe de cet objet que dans la mesure où cet objet a lui-même la nature d'un signe, de la pensée. Car le signe n'affecte pas l'objet, mais en est affecté, de sorte que l'objet doit être capable de communiquer la pensée, c'est-à-dire avoir la nature de la pensée ou d'un signe. (Ibid.: 115)

D'où il s'ensuit la distinction entre signe et representamen, qui en est une de valeur: tout signe n'est pas representamen.

J'emploie ces deux mots signe et representamen, différemment. Par signe j'entends tout ce qui communique une notion définie d'un objet de quelque façon que ce soit, étant donné que ces communications de pensée nous sont familières. Partant de cette idée familière, je fais la meilleure analyse que je peux de ce qui est essentiel à un signe et je définis un representamen comme étant tout ce à quoi cette analyse s'applique. En conséquence, si j'ai commis une erreur dans mon analyse, une partie de ce que j'ai dit des signes sera fausse. Car dans ce cas, il se peut qu'un signe ne soit pas un representamen. (Ibid.: 116-117)

Le signe est ce qui frappe la perception; mais, dans un premier temps, rien $\mathrm{n}^{\prime}$ indique encore que ce signe soit relié à un objet dont il tient lieu, par la vertu de l'objet à se faire signe. Mais une fois que cette liaison est reconnue, alors le signe devient representamen - et correspond à la définition précitée, ce qui précisément le distingue du signe «familier ». Ainsi l'on comprend cette autre définition, qui ne traite pas du signe:

2 bis. Ma définition d'un representamen est la suivante: UN REPRESENTAMEN est le sujet d'une relation triadique avec un second appelé son OBJET, POUR un troisième appelé son INTERPRÉTANT, cette relation triadique étant telle que le REPRESENTAMEN détermine son interprétant à entretenir la même relation triadique avec le même objet pour quelque interprétant. (Ibid.: 117)

Cette définition précise la précédente, entre autres en déterminant un ordre qui permet de renforcer le lien avec les Catégories; où donc le representamen est le premier d'un second, son objet, pour un troisième, l'interprétant ${ }^{12}$. On remarque ensuite le critère de validité du signe, qui est l'identité des relations entre interprétant et objet, et entre representamen et objet - puis, à un autre niveau, identité de relation pour tout autre interprétant, car il peut y en avoir plus d'un ${ }^{13}$. On notera, par ailleurs, les deux éléments qui manquent à cette autre définition et qui confirment sa «restriction»: il n'est plus fait référence à une "personne», car l'interprétant l'implicite sans y correspondre (l'interprétant est toujours «interprétant pour quelqu'un», mais n'est pas l'interprète); puis il manque encore la référence au «fondement» qu'on retrouve dans la première définition. Cette omission mérite quelque attention. Dans la suite de la citation 2, Peirce précise ce qu'il entend par «idée» («[...] idée que j’ai appelée quelquefois le fondement du representamen »); il tend à la rattacher à un sens courant qui marque toujours plus ou moins la continuité:

[...] où nous disons qu'un homme saisit l'idée d'un autre homme; où nous disons, quand un homme se souvient de ce qu'il pensait quelque temps auparavant, qu'il se souvient de la même idée; et où nous disons, quand un homme continue à penser à quelque chose [...] qu'il a la même idée [...]. (Ibid.: 121)

L'Idée serait donc une continuité transmissible sur divers individus, sur divers moments ou sur divers lieux. Le fondement, qui sert de restriction dans la définition précitée, correspondrait à cette continuité particulière au signe qui est l'effectivité de la relation, une fois reconnue. Le fondement est lié à ce «point de vue» qui permet la continuité de la relation; or, nous avons vu que c'est par le même critère qu'un signe se révélait un representamen, ce qui semble expliquer l'omission du «fondement » dans la définition 2bis, où il serait implicite.

3. Je définis un Signe comme étant quelque chose qui est si déterminé par quelque chose d'autre, appelé son objet, et qui par conséquent détermine un effet sur une personne, lequel effet j'appelle son Interprétant, que ce dernier est par là même médiatement déterminé par le premier. (Ibid. : 51)

Nous voulons ici lier deux aspects qui nous semblent particulièrement clairs dans cet extrait, quoique nous les ayons déjà rencontrés dans les autres citations: il s'agit de la relation entre l'effet et la médiation. Nous sommes amenés à penser le signe en termes d'effet, de projection, de «désadhérence», de «sortie hors de soi », cette qualité que nous avons attribuée à certains objets aptes à produire des signes ${ }^{14}$; et, ce faisant, il semble qu'un tiers soit nécessairement impliqué dans le processus et que l'effet du signe doive passer par une médiation ${ }^{15}$. 
4. [Un signe] est tout ce qui détermine quelque chose d'autre (son interprétant) à renvoyer à un objet auquel lui-même renvoie (son objet) de la même manière, l'interprétant devenant à son tour un signe et ainsi de suite ad infinitum.

Il est certain que la conscience intelligente doit entrer dans la série. Si la série des interprétants successifs s'arrête, le signe devient par là même à tout le moins imparfait. (Peirce, 1978: 126)

Cette définition, quant à elle, met en relief ce qu'on appelle la «semiosis illimitée» du signe, de même que son interprétation. $\mathrm{Si}$, comme nous l'avons précisé plus haut, chaque effet se manifeste par une médiation, $c^{\prime}$ est donc qu'un tiers est toujours impliqué dans la signification et est susceptible à son tour de développer une médiation. Par delà la continuité du fondement, la relation (effective) apportée par l'interprétant met en branle une semiosis potentiellement illimitée; et l'interprétation se jouera entre ces deux limites, entre continuité et semiosis, comme le montre bien le parcours d'Umberto Eco (de l'OEuvre ouverte aux Limites de l'interprétation). C'est aussi dire, enfin, que l'interprétation fait partie de la définition du signe, qu'elle délègue pour chaque signe un (ou plusieurs) signe(s), l'interprétant, qui le marque dans ce qu'il signifie pour quelqu'un. Peirce s'est bien défendu d'inclure l'interprète dans sa définition, ce qui aurait été bien sûr anti-scientifique ${ }^{16}$. Mais, pour nous, sa définition a ceci de particulier qu'elle est doublement dynamique: d'une part parce qu'elle établit la médiation infinie de la semiosis, I'action du signe; d'autre part parce qu'elle conçoit le signe comme un objet interprétable, et qu'ainsi elle considère aussi l'action sur le signe. C'est un des points qui rapprochent la sémiotique peircienne d'une herméneutique.

\subsection{INTERPRÉTANCE ET HERMÉNEUTIQUE}

Peirce distingue trois types d'interprétants: I'interprétant affectif, l'interprétant énergétique et l'interprétant logique, qui sont encore une fois divisés dans l'optique des trois Catégories. Le premier est "un sentiment que le signe produit» et permet souvent de reconnaître le signe pour lui-même, sans assurer cette reconnaissance cependant; le deuxième produit un effet qui «impliquera toujours un effort», physique ou mental, comme un commandement; et le troisième est un concept ou une pensée qui «a une nature générale», contrairement à l'interprétant énergétique mental qui n'est qu'un «acte singulier» (Peirce, 1978: 130). Dans le cas d'une œuvre d'art, le premier type d'interprétant représente tous les états d'âme qu'elle peut susciter. Peirce a raison de parler, dans ce cas, d'une reconnaissance soumise à caution ; I'on sait que c'est ainsi que nous apprécions communément une œuvre ( «elle m’a bouleversé, m’a transporté, m’a ému, etc. »), tout en reconnaissant que ces affirmations sont primaires et ne garantissent pas encore que l'œuvre en question soit un signe à part entière. Le deuxième type $d^{\prime}$ interprétant est commun à tous les « interprètes » qui ont pour fonction de «mettre en actes, en scène, en geste» une œuvre: les interprètes musicaux, les comédiens. Pour eux, l'œuvre est un commandement qui agit sur leur corps et leur esprit et les «contraint», en quelque sorte, à en adopter la forme. On voit bien ici la continuité de la trichotomie peircienne, qui est extrêmement fluide: ainsi, l'expression que nous donnons en exemple d'un interprétant de premier type ( «une œuvre m'a transporté... ») pourra dénoter un interprétant de deuxième type dans le cas d'une réelle réaction physique ( «...et j'ai pleuré »). De même, la transition entre un interprétant énergétique mental et un interprétant logique se fait par un passage du singulier au général, lorsqu'une pensée est suffisamment abstraite pour devenir médiane. L'interprétant logique est donc le seul qui permette de «comprendre» un signe, et c'est en toute rigueur par lui que nous devons commencer pour l'étudier.

Comme l'interprétant est un médiateur et qu'il devient luimême le signe d'un autre interprétant, il engage une semiosis illimitée. Peirce la définit au plus simple par l'interaction entre les trois parties du signe:

Mais tout ceci ne nous dit pas exactement quelle est la nature de l'effet essentiel que produit sur l'interprète la semiosis du signe, qui constitue l'interprétant logique. (Il est important de comprendre ce que j'entends par semiosis. Toute action dynamique, ou action de la force brutale, physique ou psychique, ou bien s'exerce entre deux sujets [qu'ils réagissent également I'un sur l'autre ou que I'un soit agent et l'autre patient, entièrement ou partiellement] ou bien en tout cas la résultante d'actions entre paires. Mais par "semiosis", j'entends, au contraire, une action ou influence qui est ou implique la coopération de trois sujets, tels qu'un signe, son objet et son interprétant, cette influence tri-relative n'étant en aucune façon réductible à des actions entre paires. [...]). (Peirce, 1978: 133) 17

La semiosis définit cette action médiatrice du signe, et partant, par la fenêtre de l'interprétant, la suite de relais qu'il engage pour sa compréhension. Comme le note Eco, la semiosis est reconnaissable lorsque ce signe engage un autre signe, et ainsi de suite:

Autrement dit, on a un phénomène sémiosique lorsque, à l'intérieur d'un contexte culturel donné, un objet donné peut être représenté par le terme rose et le terme rose peut être interprété parfleur rouge, 
ou par l'image d'une rose, ou par une histoire entière qui raconte comment on cultive les roses. (Eco, 1992: 239)

La semiosis d'un signe particulier est donc potentiellement illimitée et se trouve marquée par la «Continuité» de la Tiercéité. Or ce processus trouve néanmoins sa limite, quoiqu'il s'agisse d'une «limite ouverte», dans I'habitude:

Je ne nie pas qu'un concept, une proposition ou un argument puisse être un interprétant logique. J'insiste seulement sur le fait qu'il ne peut pas être l'interprétant logique final, pour la raison qu'il est luimême un signe de cette sorte de signe précisément qui a lui-même un interprétant logique. L'habitude seule, bien qu'elle puisse être signe d'une autre façon, n'est pas un signe de la façon dont ce signe dont elle est l'interprétant logique est le signe. [...] L'habitude formée délibérément par analyse d'elle-même - parce que formée à l'aide des exercices qui la nourrissent - est la définition vivante, l'interprétant logique véritable et final. Par suite, pour exprimer le plus parfaitement possible un concept que les mots peuvent communiquer, il suffira de décrire l'habitude que ce concept est calculé à produire. (Peirce, 1978: 136-137)

C'est ici que Peirce marque son "pragmatisme», en situant la limite d'un signe dans cette habitude développée «délibérément ». Ce reversement du signe dans le monde clôt le cercle de la semiosis et la «spéculation» du signe. Or, Peirce marque une concession lorsqu'il précise que l'habitude "puisse être signe d'une autre façon», ce dont Eco justement profite pour noter que la finalité $d^{\prime}$ un signe n'est que la naissance d'un autre signe:

Dans cette perspective, le cercle de la semiosis se ferme à chaque instant et ne se ferme jamais. Le système des systèmes sémiotiques, qui pourrait apparaître, de façon idéaliste, comme un univers culturel séparé de la réalité, amène en fait à agir sur le monde et à le modifier; mais chaque action modificatrice se convertit à son tour en signe et donne naissance à un nouveau processus sémiosique.

(Eco, 1985: 54)

Il faudrait encore noter le lien qui unit l'habitude et la croyance, qui sont en fait une seule et même chose: " [être prêt] à agir d'une certaine façon dans des circonstances données et quand on y est poussé par un mobile donné, voilà ce qu'est une habitude; et une habitude délibérée ou auto-contrôlée est précisément une croyance» (Peirce, 1978: 132). Pour Peirce, la croyance est ce qui règle les actions de I'humain, elle est perceptible par l'habitude qu'elle provoque, lorsque celle-ci marque une adhésion à une cause, c'est-à-dire à un concept, à une pro- position ou à un argument, donc à un signe au sens large où Peirce l'entend. Cette croyance en un signe est I'habitude qui constitue la limite de ce même signe; nulle part mieux qu'ici voit-on le cercle infini de la semiosis, ce qui permet à Peirce d'avancer ceci :

À première vue, il est, semble-t-il, paradoxal d'énoncer que "l'objet de la croyance finale qui n'existe qu'en conséquence de la croyance doive lui-même produire la croyance»; mais il y a eu un grand nombre de cas où nous avons adopté une conception de l'existence semblable à celle-ci. L'objet de la croyance n'existe, il est vrai, que parce que la croyance existe; mais ce n'est pas la même chose que de dire qu'elle commence à exister au moment où la croyance commence à exister. (Peirce, 2002 : 181-182)

La croyance serait ainsi par excellence une pensée prédicative, qui agit en fonction d'un objet qu'elle ne peut que présupposer dans un premier temps. Or, ce principe est à la base d'une certaine herméneutique, entre autres lorsque Gadamer défend certains «préjugés » (1996: 298-312); la compréhension s'effectue après une espèce de pré-compréhension, une intuition qui guide l'entendement vers une fin qu'elle devine. Et la compréhension sera ce processus par lequel on retrouvera cette fin (souvent modifiée ou déplacée, il va sans dire) qui l'aura motivée.

Rappelons encore ici ce qu'Eco notait plus haut: que ce cercle n'est pas fermé sur lui-même, mais qu'il introduit au contraire le monde dans son rayon. Le signe peircien n'est pas cantonné dans un mensonge ou une fiction qui en réduirait l'action; au contraire, il se définit par ses effets, et Peirce n'hésite pas à affirmer que les pensées et les émotions sont aussi réelles que les faits. Il rattache explicitement l'interprétant à une action sur le réel, à un "événement», comme le souligne Gérard Deledalle dans son commentaire:

"Suivant notre second principe qu'il n'y a pas d'intuition ou de connaissance qui ne soit déterminée par des connaissances antérieures, écrit ailleurs Peirce, il suit que l'apparition d'une nouvelle expérience n'est jamais une affaire instantanée, mais est un événement occupant du temps et venant à passer par un processus continu». (5.284)

Syntactiquement, il serait absurde d'exiger la réalisation complète de toutes les séries d'événements interprétants pour interpréter un signe; pragmatiquement, l'événement réalise d'une manière continue cette interprétation dans le temps. Le signe interprétant n'est pas absolument identique au signe qui le produit: il peut être un signe «équivalent» ou "plus développé » (2.228); il peut être aussi 
ce signe plutôt que tel autre-mais ce choix ne se fait pas au hasard.

(Peirce, 1978: 220)

Le signe peircien est ainsi posé comme objet susceptible d'intéresser l'herméneutique, lorsqu'il « comprend » dans son sens I'interprétant qui le fait advenir et lorsqu'il suscite à sa limite une habitude qui est une croyance. II reste encore à donner trois aspects du signe peircien qui ouvrent sur les voies de l'herméneutique et qui semblent confirmer la valeur d'un rapprochement des deux disciplines: a) la polysémie, b) la prescription et c) l'adresse.

a) Antoine Compagnon note bien comment le signe peircien augmente le signe saussurien, non seulement parce qu'il introduit dans sa définition un troisième terme, mais bien plutôt parce que ce troisième terme est lui-même pluriel, «relationnel » :

En somme, le signe de Peirce et celui de l'ancienne rhétorique, auxquels la citation serait conforme, sont bien des relations, mais non binaires: plurielles (on parlera des relata du signe ou de la citation, qui sont plus de deux). Ce sont, pour opposer le concept mathématique de correspondance, à celui de relation, des choses qui renvoient à plusieurs, à diverses autres choses et qui transportent du et des sens, comme on peut circuler à travers tout Paris avec un seul signe en poche au départ, un seul billet de métro, si l'on emprunte les correspondances. (Compagnon, 1979: 65)

C'est pourquoi le signe peircien se donne d'emblée pour être polysémique, ce qui n'implique nullement qu'il pose une polysémie «légale», colligée dans les dictionnaires; seulement, comme le signe est toujours perçu pour quelqu'un qui lui fournit ses interprétants propres, ceux-ci peuvent être aussi divers que la relation triadique le supportera ${ }^{18}$. C'est aussi dire que la polysémie du signe peircien est foncièrement «fonctionnelle » et que, si les interprétants sont multiples, c'est entre autres parce qu'ils sont de divers ordres: "L'interprétant peut être une paraphrase, une inférence, un signe équivalent appartenant à un système de signes différent, un discours entier, et ainsi de suite» (Eco, 1992 : 239). Pour Eco, chaque signe développe sa propre encyclopédie que le « lecteur modèle » aura comme tâche d'actualiser. La valeur du signe peircien est ainsi déplacée, d'un dogmatisme du sens, de sa fixité, vers sa circulation, ses relations engendrées, la valeur fixe étant plutôt, si l'on veut, la «continuité » soutenue dans un tel processus. Ainsi Eco, par son allégeance peircienne, situe la polysémie du côté de la demande du signe, plutôt que du côté d'une supposée liberté du lecteur: «Entendre un signe comme une règle qui se développe à travers la série de ses propres inter- prétants signifie avoir acquis I'habitude d'agir selon la prescription fournie par le signe» (Eco, 1985: 53) ${ }^{19}$.

b) Comme le dit Eco, dans l'extrait que nous venons de citer, tout signe a sa "prescription», qui est sa continuité 20 . Relever le signe, l'étudier, l'interpréter, c'est toujours plus ou moins poursuivre son "enchaînement», son commandement. II ne s'agit pas là d'une donnée éthique, qui engagerait une soumission de I'interprète, mais d'une donnée pleinement sémiotique: la volonté qu'aura l'interprète de poursuivre l'enchaînement du signe le fait vivre et évoluer. De même que l'objet se fait signe par sa qualité à se projeter, de même le signe se projette dans l'interprétant et commande certaines acceptions plutôt que d'autres, certains usages, certaines réactions. L'interprète n'est pas lésé dans son pouvoir par ce commandement, bien au contraire; car $c^{\prime}$ est en respectant les commandements du signe qu'il pourra mieux le saisir, le comprendre, et ainsi s'en donner la maîtrise ${ }^{21}$. À l'opposé, l'interprète aura toujours du mal à tenir un signe qu'il « dévie», qu'il détourne dans ses intérêts propres; ce manque de continuité du signe pourra avoir des effets pervers, et l'on sait que parfois les mots peuvent se retourner contre les sujets qui les manient. Et l'on sait, à l'inverse, quel effet positif peut rejaillir sur la personne qui présente un signe pleinement continu; ainsi, de l'interprète musical qui voit toute sa valeur tenir dans sa réceptivité, son «écoute», on dit parfois son « effacement», et qui lui permet de redonner le signe de notation tel qu'il l'a recueilli. Respecter le commandement du signe est donc un facteur "d'autorité», au sens plein: à la fois impératif et créateur.

c) Cela s'explique encore du fait que la négociation inaugurée par le signe en est une de l'adresse. Un signe, toujours, s'adresse à; ce qui donne toute sa richesse à l'interprétant peircien, qui intègre à même le schéma du signe une relation d'adresse. On ne peut manier efficacement un signe en faisant abstraction du fait qu'il s'adresse à quelqu'un et en se dérobant d'une écoute et d'un accueil que le signe réclame. Car c'est la tournure propre au signe de se projeter hors de lui-même pour entamer un échange avec les autres signes, une «correspondance » avec les interprètes. En même temps, cette relation est sélective et doit répondre à certains critères pour devenir effective; un critère " $d$ 'adresse » en quelque sorte, ce que rendait la deuxième définition du terme évoquée au tout début (qualité d'une manœuvre qui atteint son but). Ce critère sélectif, nous l'avons vu à l'œuvre dans les définitions de Peirce, lorsqu'il posait comme restriction, par exemple, qu'un representamen se révélait «au terme de I'analyse». Le signe, qui marque la continuité de ses constituants, devient apte à entrer en relation avec l'extérieur. En ce sens, la 
limite du signe est le «quelqu'un» qui le reçoit, celui qui en fournit les interprétants. Sans cette adresse, le signe ne survit plus et devient lettre morte. C'est parce qu'il est représentation, projection de lui-même, que le signe frappe un public, des récepteurs. Et engager un public dans son giron, ce n'est toujours que faire intervenir un tiers dans une relation binaire; car dès qu'on a le tiers, on a la «possibilité » du tiers, c'est-à-dire une fenêtre ouverte sur l'inconnu, un interprétant.

\section{LA FIDUCIE DU SIGNE}

Le signe peircien est donné d'avance. Il représente une monnaie qui s'acquiert sans rétribution, mais qui par le travail $d^{\prime}$ un individu produit une valeur fiduciaire. Il est ainsi saisi, non comme quelque chose qui commence et progresse, mais plutôt comme une force déjà commencée et prête à prendre les tangentes. En cela il interpelle directement I'herméneutique, qui représente cette écoute qui permet d'accomplir le signe peircien. Il l'interpelle comme science, mais il interpelle également l'individu récepteur, de façon très pragmatique; le signe recèle une demande, une offre aussi, une gageure à laquelle est appelé à répondre l'individu qui le reçoit. Le signe va vers son récepteur, le cherche, le crée même, le prévoit et le demande, puisqu'il l'a déjà inscrit dans ses filets, lorsqu'il commençait à s'énoncer. Ainsi, lire le signe est toujours une façon de se retrouver, de percevoir mon bien propre dans cela qui passe et s'offre ouvert à mon intervention. Je suis la limite par où le signe commence.

\section{NOTES}

1. Pour l'opposition à I'herméneutique, voir Fisette (1996: 38-44).

2. C'est la matière des premières pages du premier chapitre de I'Introduction à l'étude des textes (1995: 31-59). Ainsi, « Pour conclure: I'unité du texte n'est jamais que la projection de la cohérence de l'analyse. Du moins ne peut-on pas prouver qu'il en est autrement. Petit corollaire pour l'analyse, sur lequel on reviendra: on considérera que la meilleure analyse est non pas celle qui rend le mieux compte de l'unité du texte, mais celle qui est la plus élégante, celle qui, à partir du plus petit nombre d'hypothèses, rend compte du plus grand nombre de faits » (ibid.: 58).

3. Gadamer (1996). Voir, entre autres, p. 174 : «Cette destination appartient à leur [les œuvres esthétiques] être même parce que leur être est représentation »; et p. 417-418: « Nous avons vu à quel point la littérature se définit par la volonté de transmettre. Mais celui qui transcrit et transmet le fait lui aussi pour ses propres contemporains. Ainsi, la référence au lecteur originel tout comme la référence au sens donné par l'auteur ne semblent représenter qu'une règle herméneutique et historique très rudimentaire, à laquelle il n'est pas vraiment permis de limiter l'horizon de sens de textes. Ce qui est fixé par écrit s'est détaché de la contingence de son origine et de son auteur et s'est libéré positivement pour contracter de nouvelles relations .

4. Notre texte, en fait, divise cette deuxième partie en 1.2 et 1.3 , l'interprétant, d'une part, et l'interprétance, d'autre part. Nous avons procédé ainsi pour mettre en lumière le "pont», la médiation de l'interprétant, de même que son «action", son mouvement.

5. Peirce (2002: 31): «À la lumière des faits externes, les seuls cas de pensée que nous puissions trouver sont des cas de pensées par signes. II est clair qu'on ne peut avoir de preuves d'aucune autre pensée par des faits externes. Mais nous avons vu que c'est seulement par les faits externes que I'on peut connaître la pensée. Donc la seule pensée dont il soit possible d'avoir la cognition est la pensée par signes. Mais la pensée dont on ne peut avoir la cognition n'existe pas. Toute pensée, par conséquent, doit nécessairement être en signes».

6. Ibid.: « De la proposition que toute pensée est un signe, il découle que toute pensée doit s'adresser à une autre pensée, qu'elle doit en déterminer une autre, puisque telle est l'essence du signe» (je souligne).

7. L'autre dérive étant $d^{\prime}$ omettre généralement le «référent» du signe saussurien. Mais on verra plus loin que, même s'il fallait considérer une " trichotomie » du signe saussurien, Peirce propose encore une expansion car son signe est « relationnel » plutôt que « correspondant » (ibid. : 14-15). 8. Voir, entre autres, cet extrait: « Une chose considérée pour elle-même est une unité. Une chose considérée comme corrélation ou dépendance ou comme effet, est seconde par rapport à quelque chose d'autre. Une chose qui, de quelque façon, met une chose en relation avec une autre est troisième ou placée au milieu des deux autres » (The Essential Peirce, trad. par J. Fisette, 1996: 250).

9. Collot (1997: 46-47): «Il faut donc opérer une sorte de révolution copernicienne, par laquelle le sujet, au lieu d'imposer au monde ses valeurs et ses significations préétablies, accepte de se "transférer aux choses", pour découvrir en elles "un million de qualités inédites", qu'il pourra s'approprier, s'il parvient à les formuler». Les citations sont extraites de Proêmes de Ponge.

10. Peirce (1978: 74): «Premier et second, agent et patient, oui et non, sont des catégories qui nous permettent de décrire en gros les faits de l'expérience, et l'esprits'en est contenté pendant longtemps. Mais enfin on les a trouvées inadéquates et l'on a fait alors appel à une autre conception, au troisième. Le troisième est ce qui jette un pont sur l'abîme entre le premier et le dernier absolus et les met en relation ».

11. Peirce (1978: 115): «Or dans la tiercéité authentique, le premier, le 
second et le troisième ont tous trois la nature de troisièmes ou de la pensée, alors que par rapport l'un à l'autre ils sont premier, second et troisième ». 12. Ainsi plus haut, dans nos définitions des Catégories, la Qualité (Priméité) recevait un caractère de liaison lorsque la définition la présentait au terme d'une analyse. De la même façon ici, le representamen prend place du «signe» lorsque celui-ci, au terme d'une analyse, révèle ses liaisons effectives et dépasse la seule perception brute (Priméité).

13. Ce critère d'identité est encore plus explicite dans cette autre définition: "Un signe est donc un objet qui est en relation avec son objet d'une part et avec un interprétant d'autre part, de façon à mettre l'interprétant en relation avec cet objet, correspondant à sa propre relation avec cet objet » (ibid.: 30).

14. C'est, si l'on veut, la « désadhérence » du signe, son abandon, son autonomie, sa faculté à conserver une relation à l'objet mais en dehors de lui; d'où, encore, sa « répétabilité » : «Le mode d'être d'un representamen est de nature à être susceptible de répétition » (Peirce, 2002: 377).

15. Voir cet autre extrait: «Or la Tiercéité n'est que le caractère d'un objet qui incarne l'Entre-deux-ité (Betweenness) ou la Médiation sous sa forme la plus simple et la plus rudimentaire; et je l'emploie pour désigner cet élément du phénomène qui est prédominant partout où la Médiation est prédominante, et qui atteint sa plénitude dans la Représentation » (ibid. : 347 ; je souligne).

16. Voir cette autre définition, où il est tout près de l'inclure: « Un signe est un lieu virtuel de connaissance [Cognizable] qui, d'un côté est déterminé (c'est-à-dire spécifié, bestimmt) par quelque chose d'autre que lui-même, appelé son objet alors que, d'un autre côté, il détermine quelque esprit actuel ou potentiel ; cette détermination, je l'appelle l'interprétant créé par le signe de sorte que l'esprit qui interprète est médiatement déterminé par l'objet» (8.177, dans Fisette, 1996: 267).

17. Le passage entre crochets est introduit par le traducteur.

18. Compagnon (1979: 61): «Ce qui fait le trois dans cette formule, c'est somebody, quelqu'un: il n'y a pas de signe sans quelqu'un à qui il fait signe. II n'y a de signe que pour quelqu'un ".

19. M. Charles, d'un point de vue complètement différent, relève le même aspect: «Une relation figurale implique un geste du lecteur, comporte une intervention critique. À chaque fois une décision est à prendre. Est-ce à dire que cette décision est libre? Évidemment non : un texte est fait (aussi) de trous et de failles où intervient, où se place l'interprétation; mais ces trous et ces failles sont marqués, signalés. Et à supposer que la décision soit libre, c'est (encore) un effet du texte» (1977: 118).

20. Voir l'extrait 2.230, dans Peirce (1978: 122-123) : «En conséquence, tout signe a, en acte ou virtuellement, ce que nous pouvons appeler un précepte d'explication suivant lequel il faut le comprendre comme étant, pour ainsi dire, une sorte d'émanation de son objet ».

21. Ce lien, cette imbrication de la prescription et de la liberté a bien été établie parM. Charles, lorsqu'il traite de "l'autorité » et du " commentaire ": " Nous voici maintenant avec deux définitions du texte: un texte est un être de langage qui fait autorité; un texte est ce qui faitl'objet d'un commentaire. Il est clair que la seconde définition comprend, à tous les sens du terme, la première. Le commentateur étant un être profondément respectueux par essence, la seconde définition explique en effet cette autorité qui s'attache au texte: en d'autre $[s i c]$ termes, cette définition est tout simplement une analyse critique de la première. Au lieu de dire que le texte a une autorité ou, plutôt, au lieu de me comporter constamment comme s'il en avait une, je constate que c'est moi, lecteur ou critique, qui la lui attribue». II continue d'ailleurs en prétendant que c'est le commentaire qui fait exister le texte (puisque le texte fait l'objet d'un commentaire), ce qui reviendrait à dire, en termes peirciens, que $c^{\prime}$ est l'interprétant qui donne vie au signe. (Charles, 1995: 47-48.)

\section{RÉFÉRENCES BIBLIO G RAPHIQ UES}

ChARLES, M. [1995] : Introduction à l'étude des textes, Paris, Seuil, coll. "Poétique»;

[1977] : Rhétorique de la lecture, Paris, Seuil, coll. « Poétique ». COLlot, M. [1997] : La Matière-émotion, Paris, PUF, coll. "Écriture». Compagnon, A. [1979]: La Seconde Main ou le travail de la citation, Paris, Seuil.

ECO, U. [1992] : Les Limites de l'interprétation, trad. de l'italien par M. Bouzaher, Paris, Grasset, coll. "Le Livre de Poche / Biblio / Essais »; [1985] : Lector in fabula. Le rôle du lecteur, trad. de l'italien par M. Bouzaher, Paris, Grasset, coll. "Le Livre de Poche / Biblio / Essais ». FISETTE, J. [1996] : Pour une pragmatique de la signification, suivi d'un choix de textes de C. S. Peirce en traduction française, Montréal, XYZ Éditeur, coll. «Documents».

GADAmer, H.-G. [1996]: Vérité et Méthode. Les grandes lignes d'une herméneutique philosophique, éd. intégrale revue et complétée par $\mathrm{P}$. Fruchon, J. Grondin et G. Merlio, Paris, Seuil, coll. «L'ordre philosophique ». PeIRCE, C. S. [2002] : Pragmatisme et Pragmaticisme, sous la dir. de C. Tiercelin et P. Thibaud, Paris, Cerf, coll. «Passages»;

[1978] : Écrits sur le signe, rassemblés, traduits et commentés par

G. Deledalle, Paris, Seuil, coll. «L'ordre philosophique». 